\title{
Detection of Haemophilus parasuis isolates from South China by loop-mediated isothermal amplification and isolate characterisation
}

\author{
Authors: \\ Jian-min Zhang \\ Hai-yan Shen ${ }^{1,2}$ \\ Ming Liao \\ Tao Ren ${ }^{1}$ \\ Li-li Guo ${ }^{1}$ \\ Cheng-gang $\mathrm{Xu}^{1}$ \\ Sai-xiang Feng \\ Hui-ying Fan \\ Jing-yi $\mathrm{Li}^{1}$ \\ Ji-dang Chen ${ }^{1}$ \\ Bin Zhang
}

\section{Affiliations:}

${ }^{1}$ The Key Laboratory of

Animal Disease Control and Prevention of the Ministry of Agriculture, South China Agricultural University, China

${ }^{2}$ Department of

Biotechnology Research,

Veterinary Research Institute

of Guangdong Academy of

Agricultural Sciences, China

Correspondence to:

Cheng-gang $\mathrm{Xu}$

Email:

chgangxu@126.com

Postal address:

483 Wushan Road, Tianhe

District, Guangzhou city,

Guangdong Province, China

Dates:

Received: 05 Oct. 2011

Accepted: 13 Dec. 2011

Published: 24 Apr. 2012

How to cite this article: Zhang, J., Shen, H., Liao, M., Ren, T., Guo, L., Xu, C. et al., 'Detection of Haemophilus parasuis isolates from South China by loop-mediated isothermal amplification and isolate characterisation, Onderstepoort Journal of Veterinary Research 79(1), Art. \#383, 6 pages. http:// dx.doi.org/10.4102/ojvr. v79i1.383

(C) 2012. The Authors. Licensee: AOSIS OpenJournals. This work is licensed under the Creative Commons Attribution License.
Haemophilus parasuis is the etiological agent of Glässer's disease, which is characterised by fibrinous polyserositis, meningitis and polyarthritis, causing severe economic losses to the swine industry. In this study, a loop-mediated isothermal amplification (LAMP) test was developed to improve the specificity, facility and speed of diagnosis of $H$. parasuis isolates. The LAMP assay rapidly amplified the target gene within $50 \mathrm{~min}$ incubation at $63{ }^{\circ} \mathrm{C}$ in a laboratory water bath. The LAMP amplicon could be visualised directly in the reaction tubes following the addition of SYBR Green I dye. The detection limit of this LAMP method was $10 \mathrm{CFU} / \mathrm{mL}$, which was 10 times more sensitive than the earlier 16S rRNA polymerase chain reaction (PCR) test conducted by Oliveira, Galina and Pijoan (2001), and no cross-reactivity was observed from other non- $H$. parasuis strains. This LAMP test was evaluated further on 187 clinical specimens from pigs suspected of being infected with $H$. parasuis. Forty-three were found positive by bacterial isolation of $H$. parasuis, as well as by the 16S rRNA PCR and LAMP tests. The $43 \mathrm{H}$. parasuis isolates were classified into 9 serovars and had 37 genetic patterns when analysed by pulsed-field gel electrophoresis (PFGE). This displayed that various $H$. parasuis serovars and genotypes were widely distributed in South China. Therefore, the speed, specificity and sensitivity of the LAMP test, the lack of a need for expensive equipment, and the visual readout showed great potential for a correct clinical diagnosis of $H$. parasuis in favour of controlling Glässer's disease.

\section{Introduction}

Haemophilus parasuis is the causative agent of Glässer's disease in swine, a disease characterised by fibrinous polyserositis, meningitis and polyarthritis (Li et al. 2009; Olvera, Segalés \& Aragón 2007). In recent years, diseases in swine caused by H. parasuis have attracted worldwide attention (Baumann \& Bilkei 2002). It is therefore necessary to carry out timely and effective monitoring of $H$. parasuis to identify strains circulating between farm animals as a means of defining the epidemiology and types of $H$. parasuis strains present in respective areas in order to control and prevent outbreaks.

Haemophilus parasuis is a slow-growing, fastidious organism with specific nutritional requirements, making its isolation difficult (Oliveira, Galina \& Pijoan 2001). Moreover, detection of $H$. parasuis using conventional methods, including culture, biochemical or immunological assays is not effective and requires several days to complete (Angen et al. 2007). The polymerase chain reaction (PCR) provided a major advance in the diagnosis of H. parasuis infections (Oliveira et al. 2001), including nested PCR (Jung et al. 2004) and real-time PCR assays (Turni, Pyke \& Blackall 2009). However, PCR assays are limited by the equipment required to carry out the reactions and the need for skilled personnel. Fortunately, a promising candidate for a new detection method is loop-mediated isothermal amplification (LAMP), which amplifies DNA with high specificity, efficiency and rapidity under isothermal conditions. It is faster, more specific and easier to perform than conventional PCR (Nagamine, Hase \& Notomi 2002; Notomi et al. 2000). The development of LAMP procedures has been reported for many different clinical applications: the diagnosis of viral and bacterial infections (Iwamoto, Sonobe \& Hayashi 2003; Minami et al. 2006; Song et al. 2005; Wang et al. 2009), for diagnosis of protozoal diseases (Kuboki et al. 2003; Thekisoe et al. 2005) and for canine and equine piroplasmosis (Alhassan et al. 2007; Ikadai et al. 2004). The infB gene of $H$. parasuis has been proven to be useful as a genetic marker for phylogenetic studies (Turni et al. 2009), making it a better alternate gene to the 16S rRNA gene in establishing the LAMP method.

To date, $15 \mathrm{H}$. parasuis serovars have been recognised using immunodiffusion tests with heatstable antigens. However, about $25 \%$ of isolates are non-typable using this methodology (Vanier et al. 2006). In view of the fact that serotyping does not provide enough discrimination of $H$. parasuis 
isolates, an optimised and standardised pulsed-field gel electrophoresis (PFGE) method established by our research team was used to characterise the $H$. parasuis isolates. This method provides a higher discriminatory power, allowing for the typing of previously undefined isolates (Zhang et al. 2011).

In the present study, 15 serovar reference strains were used to develop the LAMP assay of $H$. parasuis. In addition, the $H$. parasuis strains isolated from clinical specimens collected from South China, were serotyped and characterised by PFGE patterns.

\section{Materials and methods}

\section{Bacterial strains}

The test was optimised using the 15 reference strains for the different serovars of $H$. parasuis and the field isolates of Actinobacillus pleuropneumoniae (serovar 5), A. pleuropneumoniae (serovar 9), two strains of Bordetella bronchiseptica, Pasteurella multocida, Streptococcus suis, Salmonella typhimurium and Escherichia coli.

\section{Clinical samples}

From 2008 to 2010, 187 specimens, including lung, nasal swab, synovia and heart from pigs suspected of being infected with H. parasuis were collected in South China.

\section{Bacterial isolation}

All 187 specimens were cultured on tryptic soy agar (OXOID Inc., Basingstoke, UK) containing $10 \mathrm{mg} / \mathrm{mL}$ nicotinamide adenine dinucleotide (NAD, Sigma-Aldrich, St. Louis, USA) and $5 \%$ bovine serum (Gibco ${ }^{\circledR}$, Invitrogen, Carlsbad, USA) for isolation of $H$. parasuis strains. Plates were incubated at $37^{\circ} \mathrm{C}$ for $24 \mathrm{~h}-72 \mathrm{~h}$ and presumptive small and translucent bacterial colonies were selected for further characterisation by PCR, LAMP and biochemical testing.

\section{DNA extraction}

Template DNA used for LAMP and 16S rRNA PCR were prepared as follows: bacterial cells of each strain from colonies on Trypticase Soy agar (TSA) were re-suspended in double-distilled water $\left(\mathrm{ddH}_{2} \mathrm{O}\right)$ to achieve a concentration of approximately $10^{6} \mathrm{CFU} / \mathrm{mL}$. In order to examine the detection limit for LAMP and 16S rRNA PCR, a 10-fold dilution series of $H$. parasuis of serovar 5 with $\mathrm{ddH}_{2} \mathrm{O}$ was made. The cells were heat-treated in a boiling water bath for $10 \mathrm{~min}$ and were centrifuged at $12000 \mathrm{rpm} / \mathrm{min}$ for $10 \mathrm{~min}$. The resulting supernatant was used as the template for the LAMP and 16S rRNA PCR.

\section{Development of the loop-mediated isothermal amplification and polymerase chain reaction methods}

Four primers corresponding to FIP, BIP, F3 and B3 (Table 1) were used for the LAMP assay and designed based on the conserved regions of the infB gene sequence (GeneBank accession numbers: EF424388), according to criteria established previously (Notomi et al. 2000; Tomita et al. 2008). The reaction mixture consisted of $0.8 \mu \mathrm{M}$ of each FIP and BIP primers, $0.2 \mu \mathrm{M}$ of each F3 and B3 outer primers, $400 \mu \mathrm{M}$ of each dNTP (Takara Bio Inc., Shiga, Japan), $1 \mathrm{M}$ betaine (Sigma-Aldrich, St. Louis, USA), 1X thermopol buffer (New England Biolabs Inc., Ipswich, USA), $2 \mathrm{mM} \mathrm{MgSO}_{4^{\prime}}$ $8 \mathrm{U}$ of Bst DNA polymerase large fragment (New England Biolabs Inc., Ipswich, USA) and $2 \mu \mathrm{L}$ of genomic DNA. The reaction mixture was incubated at $61{ }^{\circ} \mathrm{C}, 62{ }^{\circ} \mathrm{C}, 63^{\circ} \mathrm{C}$, $64^{\circ} \mathrm{C}$ or $65^{\circ} \mathrm{C}$ using a heat block for $20 \mathrm{~min}, 30 \mathrm{~min}, 40 \mathrm{~min}$, $50 \mathrm{~min}$ or $60 \mathrm{~min}$, respectively. Reactions were terminated by increasing the temperature to $80{ }^{\circ} \mathrm{C}$ for $4 \mathrm{~min}$. To determine the sensitivity of the LAMP assay, different dilutions of H. parasuis from $1 \times 10^{6} \mathrm{CFU} / \mathrm{mL}$ to $1 \times 10^{1} \mathrm{CFU} / \mathrm{mL}$ (i.e. a series of 10 -fold dilutions) was prepared and compared with PCR by using the same templates at identical concentrations. The details of the primers (Table 1) and conditions for the PCR assay for the detection of $H$. parasuis were carried out as described previously (Oliveira et al. 2001). Cross-reactivity between the primers used in LAMP PCR with DNA isolated from A. pleuropneumoniae, B. bronchiseptica, P. multocida, S. suis, S. typhimurium and E. coli was also carried out. Genomic DNA extracted from the $15 \mathrm{H}$. parasuis reference strains were used as positive controls and distilled water was used as a negative control.

\section{Detection of Haemophilus parasuis using loop-mediated isothermal amplification and polymerase chain reaction}

Detection of the LAMP reaction products was performed using 2\% agarose gel electrophoresis prepared in $0.5 \mathrm{X}$ tris-borate-EDTA buffer stained with $0.5 \mathrm{~g} / \mathrm{mL}$ ethidium bromide. Banding patterns were examined visually under natural or UV light following the addition of $1 \mu \mathrm{L}$ SYBR Green I dye to the reaction tube. PCR products were subjected to electrophoresis on a $2 \%$ agarose gel and visualised as above.

\section{Serotyping of Haemophilus parasuis isolates}

Serotyping of $H$. parasuis isolates using gel diffusion (GD) and indirect hemagglutination assays (IHA) were performed as described previously (Cai et al. 2005; Tadjine et al. 2004) and all isolates were tested using GD first. If a definitive serotype could not be determined, respective isolates were then examined by IHA testing. Isolates that could not be identified by either test were defined as non-typable (serovar NT).

TABLE 1: Loop-mediated isothermal amplification (LAMP) primers designed for detection of the Haemophilus parasuis infB gene.

\begin{tabular}{ll}
\hline Primer & Sequence (5'-3') \\
\hline P1 & 5'-GTG ATG AGG AAG GGT GGTGT-3' \\
P2 & 5'-GGC TTC GTC ACC CTC TGT-3' \\
F3 & 5'- CGATTGTGGTTGCGGTAAAC-3' \\
B3 & 5'- TAAGATTCGATAACCACACCGC-3' \\
FIP (F1c + F2) & 5'-GAACATCACCACCGAATTTCTCAGAA \\
BIP (B1C + B2) & TTTTAAACCAGAAGCAAACCCAGAGC-3' \\
\hline
\end{tabular}




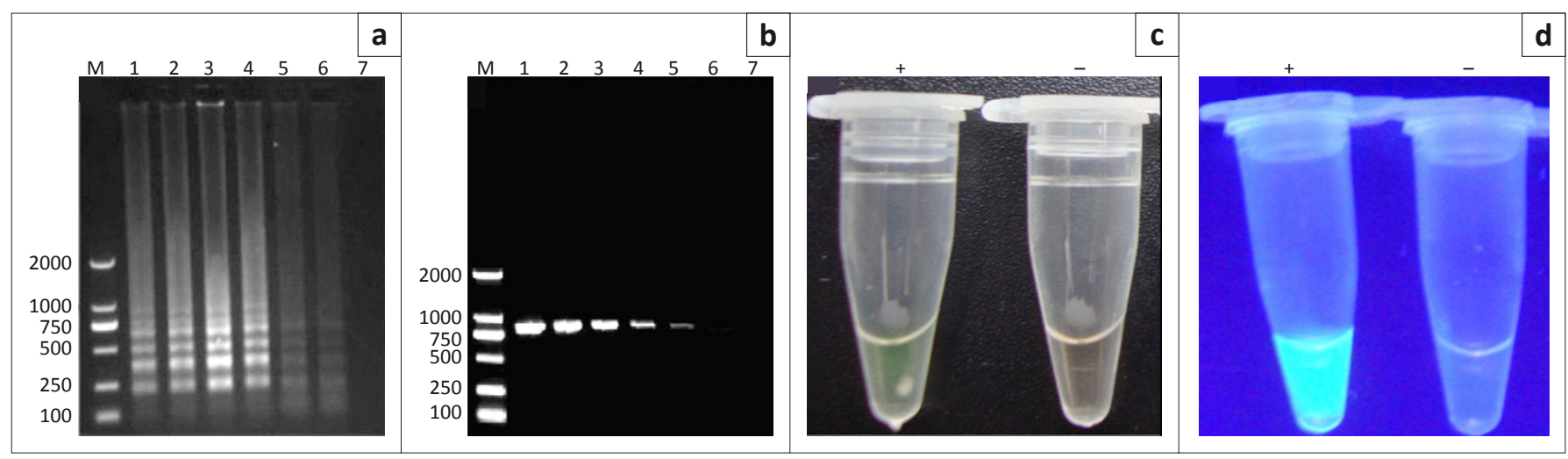

Lane M, DNA marker; Lanes $1-6,1 \times 10^{6} \mathrm{CFU} / \mathrm{mL}, 1 \times 10^{5} \mathrm{CFU} / \mathrm{mL}, 1 \times 10^{4} \mathrm{CFU} / \mathrm{mL}, 1 \times 10^{3} \mathrm{CFU} / \mathrm{mL}, 1 \times 10^{2} \mathrm{CFU} / \mathrm{mL}$ and $1 \times 10^{1} \mathrm{CFU} / \mathrm{mL}$ of Haemophilus parasuis used as a reaction template respectively; Lane 7 , negative control; +, positive reaction; -, negative reaction.

FIGURE 1: Detection of (a) infB loop-mediated isothermal amplification (LAMP) and (b) polymerase chain reaction products, as well as visual detection of infB-LAMP products under (c) daylight and (d) ultraviolet light.

TABLE 2: Comparative analysis of Haemophilus parasuis detection by either loop-mediated isothermal amplification, polymerase chain reaction or bacterial isolation.

\begin{tabular}{|c|c|c|c|c|c|c|}
\hline \multirow[t]{2}{*}{ Result } & \multicolumn{2}{|c|}{ LAMP } & \multicolumn{2}{|c|}{ PCR } & \multicolumn{2}{|c|}{ Bacterial isolation } \\
\hline & $N$ & $\%$ & $N$ & $\%$ & $N$ & $\%$ \\
\hline Positive & 89 & 47.6 & 64 & 34.2 & 43 & 23.0 \\
\hline Negative & 98 & 52.4 & 123 & 65.8 & 144 & 76.8 \\
\hline
\end{tabular}

LAMP, loop-mediated isothermal amplification; PCR, polymerase chain reaction; $N$, number of samples.

\section{Genotyping of Haemophilus parasuis by the pulsed-field gel electrophoresis method}

Haemophilus parasuis field isolates were genotyped using the PFGE method established by our research team to determine the number of different genotypes (Zhang et al. 2011). A dendrogram illustrating the genetic relatedness between identified $H$. parasuis isolates was generated using Bionumerics 4.0 software (Applied Maths, Inc., Austin, USA), with a $1.5 \%$ tolerance for fragment shifts.

\section{Ethical considerations}

All animal experiments conducted for the purposes of this study conform to the Chinese national laws regarding the use of animals in biomedical research.

\section{Results}

\section{Rapid, sensitive and specific detection of Haemophilus parasuis using loop-mediated isothermal amplification}

The LAMP method described in this study was performed in a simple water bath at $63^{\circ} \mathrm{C}$ for $50 \mathrm{~min}$. All positive LAMP reactions produced a characteristic ladder of multiple bands and the results could also be seen after staining with SYBR Green I (Figure 1). The detection limit of LAMP was $10 \mathrm{CFU} / \mathrm{mL}$, which was 10 times more sensitive than $16 \mathrm{~S}$ rRNA PCR (Figure 1). LAMP accurately detected all 15 $H$. parasuis reference strains and no amplification products were detected when either DNA from the other swine bacterial pathogens, or the negative (no template) reaction control was used (Figure 2).

\section{Detection and isolation of Haemophilus parasuis}

A total of 187 clinical specimens were obtained from pigs in South China that were suspected of being infected with H. parasuis. Eighty-nine (47.6\%) and 64 (34.2\%) samples tested positive for $H$. parasuis by LAMP and PCR, respectively. Forty-three samples (23.0\%) were positive for $H$. parasuis by bacterial isolation and all the 43 samples tested positive for H. parasuis by PCR and LAMP (Table 2).

\section{Serotyping and genotyping of Haemophilus parasuis isolates}

Nine of the fifteen recognised serovars were identified amongst the isolates collected from South China. Serovar 5

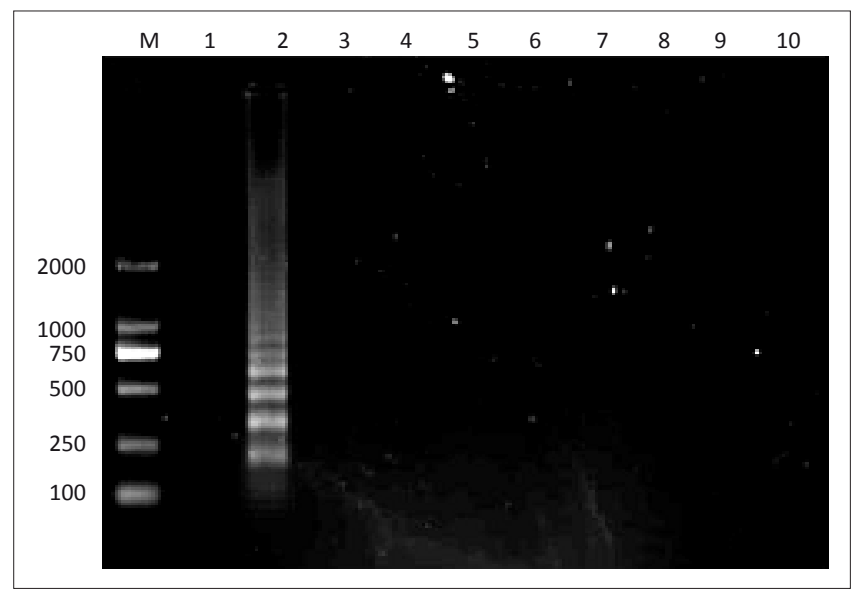

Lane M, DL2000 molecular marker; Lane 1, negative control; Lane 2, Haemophilus parasuis; Lane 3, Actinobacillus pleuropneumoniae of serovar 5; Lane 4, A. pleuropneumoniae of Lenctica serovar 9; Lane 5, Bordetella bronchiseptica strain-1; Lane 6, B. bronchiseptica strain-2; Lane 7, Pasteurella multocida; Lane 8, Streptococcus suis; Lane 9, Salmonella typhimurium; Lane
10, Escherichia coli. FIGURE 2: Specificity tests of loop-mediated isothermal amplification in agarose gel under ultraviolet light for Haemophilus parasuis detection. 


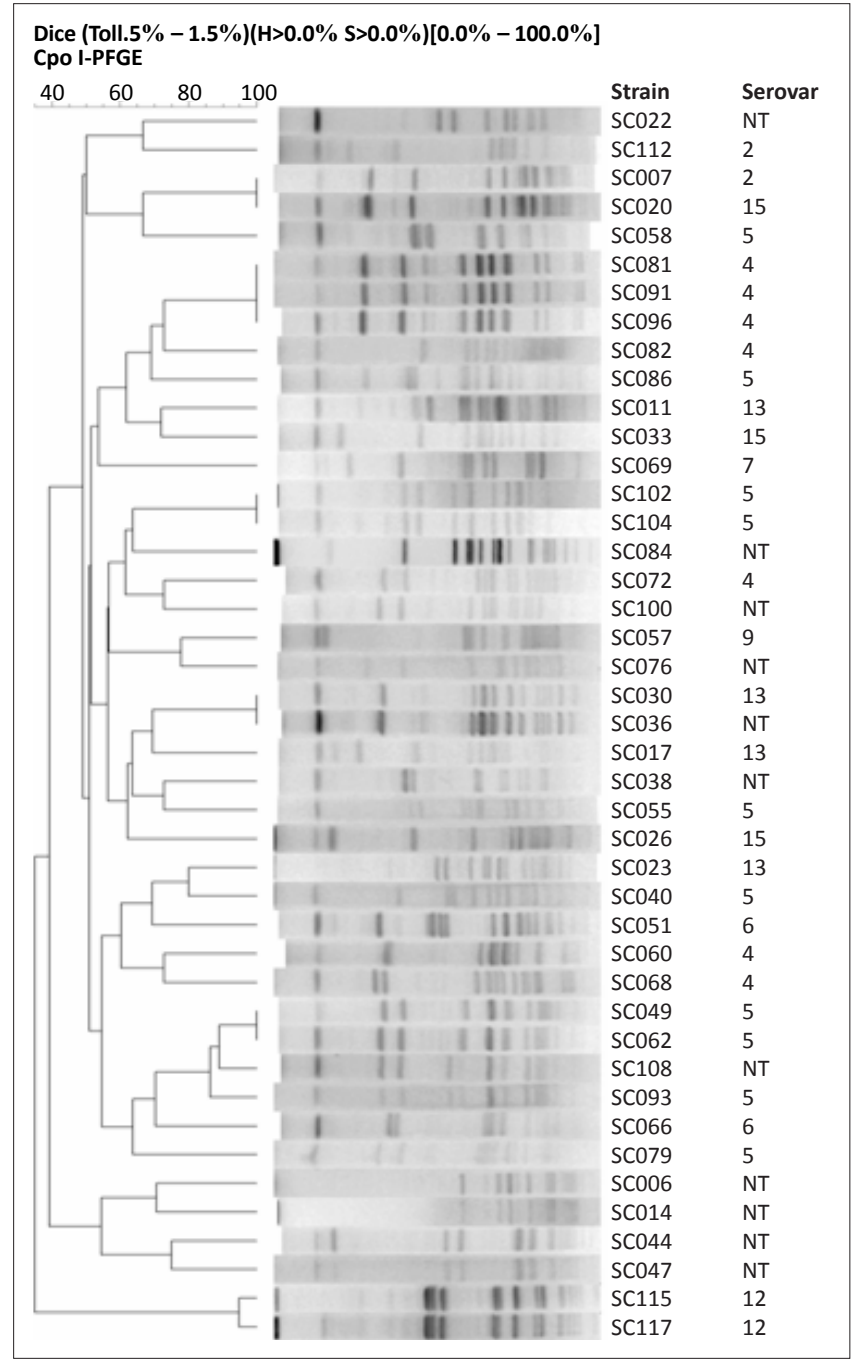

FIGURE 3: Dendrogram of pulsed-field gel electrophoresis subtype patterns of all 43 Haemophilus parasuis field strains identified.

was the most prevalent (23.3\%), followed by serovar 4 (16.3\%), whilst $25.6 \%$ of strains were non-typable by either GD or IHA. Serovars 2, 6, 7, 9, 12, 13 and 15 were represented by a small number of strains only. All $43 \mathrm{H}$. parasuis isolates were typed by PFGE and great genetic diversity was observed. A total of 37 different PFGE patterns were observed from the 43 H. parasuis isolates (Figure 3 and Table 3). In addition, strains that could not be typed by GD or IHA tests were genotyped by PFGE and displayed unique PFGE patterns. Genotyping analysis demonstrated that the $H$. parasuis population was heterogeneous and no dominant clones predominated.

\section{Discussion}

Haemophilus parasuis is a commensal of the upper respiratory tract in domestic pigs and is also the etiological agent of Glässer's disease (Oliveira et al. 2001). However, the bacterium can cause acute disease when introduced into immunologically naive herds (Little 1970). Laboratory diagnosis of $H$. parasuis using conventional methods such as culture, biochemical and immunological assays is time-consuming and laborious. Haemophilus parasuis is a fastidious bacterium and its isolation in pure culture from
TABLE 3: Characterisation of 43 Haemophilus parasuis field isolates from South China.

\begin{tabular}{|c|c|c|c|c|c|}
\hline Isolate code & Isolation site & Serovar & PFGE type & LAMP & PCR \\
\hline Sc006 & Lung & NT & $\mathrm{A} 1$ & + & + \\
\hline SC007 & Lung & 2 & $\mathrm{~A} 2$ & + & + \\
\hline SC020 & Lung & 15 & A2 & + & + \\
\hline SC011 & Nasal & 13 & A3 & + & + \\
\hline SC014 & Lung & NT & A4 & + & + \\
\hline SC017 & Synovia & 13 & A5 & + & + \\
\hline SC022 & Lung & NT & A6 & + & + \\
\hline SC023 & Synovia & 13 & A7 & + & + \\
\hline SC026 & Synovia & 15 & A8 & + & + \\
\hline SC030 & Lung & 13 & A9 & + & + \\
\hline SC036 & Nasal & NT & A9 & + & + \\
\hline SC033 & Synovia & 15 & A10 & + & + \\
\hline SC038 & Lung & NT & A11 & + & + \\
\hline SC040 & Heart & 5 & A12 & + & + \\
\hline SC044 & Lung & NT & A13 & + & + \\
\hline SC047 & Lung & NT & A14 & + & + \\
\hline SC049 & Lung & 5 & A15 & + & + \\
\hline SC062 & Heart & 5 & A15 & + & + \\
\hline SC051 & Lung & 6 & A16 & + & + \\
\hline SC055 & Synovia & 5 & A17 & + & + \\
\hline SC057 & Lung & 9 & A18 & + & + \\
\hline SC058 & Lung & 5 & A19 & + & + \\
\hline SC060 & Synovia & 4 & $\mathrm{~A} 20$ & + & + \\
\hline SC066 & Synovia & 6 & A21 & + & + \\
\hline SC068 & Lung & 4 & $A 22$ & + & + \\
\hline SC069 & Lung & 7 & A23 & + & + \\
\hline SC072 & Lung & 4 & A24 & + & + \\
\hline SC076 & Heart & NT & A25 & + & + \\
\hline SC079 & Nasal & 5 & A26 & + & + \\
\hline SC081 & Lung & 4 & $\mathrm{~A} 27$ & + & + \\
\hline Sc091 & Lung & 4 & $\mathrm{~A} 27$ & + & + \\
\hline Sc096 & Synovia & 4 & $\mathrm{~A} 27$ & + & + \\
\hline SC082 & Lung & 4 & A28 & + & + \\
\hline SC084 & Lung & NT & A29 & + & + \\
\hline SC086 & Synovia & 5 & $\mathrm{~A} 30$ & + & + \\
\hline SC093 & Lung & 5 & A31 & + & + \\
\hline SC100 & Lung & NT & $\mathrm{A} 32$ & + & + \\
\hline SC102 & Synovia & 5 & A33 & + & + \\
\hline SC104 & Nasal & 5 & A33 & + & + \\
\hline SC108 & Lung & NT & A34 & + & + \\
\hline SC112 & Lung & 2 & A35 & + & + \\
\hline SC115 & Lung & 12 & A36 & + & + \\
\hline SC117 & Lung & 12 & A37 & + & + \\
\hline
\end{tabular}

PFGE, pulsed-field gel electrophoresis; LAMP, loop-mediated isothermal amplification; PCR, polymerase chain reaction.

polymerase chain reaction.
+ , Denotes positive reaction.

diseased animals is usually difficult; therefore, rapid and accurate identification of $H$. parasuis isolates is essential for the diagnosis of Glässer's disease and a key element for controlling the disease (Aarestrup, Seyfarth \& Angen 2004; Oliveira \& Pijoan 2004). A fast and accurate LAMP test was developed to improve the diagnosis of $H$. parasuis infections. This LAMP test can be used to detect $H$. parasuis in both pure culture and clinical samples.

The LAMP method can amplify DNA with high specificity, efficiency and speed under isothermal conditions (Nagamine et al. 2002; Tomita et al. 2008). The LAMP assay also can be performed on-site, using a simple and inexpensive experimental set-up, such as a water bath or heat block that 
provides a constant temperature. In addition, the LAMP reaction products can be evaluated by a visual inspection of the colour change of the mixture when SYBR Green I dye is added. After isolation of $H$. parasuis from the clinical specimens, the LAMP can be performed as a substitute for 16S rRNA PCR tests for bacterial identification, eliminating the need for time-consuming electrophoresis and costly specialised equipment, as well as reducing the time for the diagnosis of $H$. parasuis infection considerably.

It has been reported that the $16 \mathrm{~S}$ rRNA gene is not speciesspecific to enable $H$. parasuis to be distinguished from other closely related species when the short amplification sequence of real-time PCR was used (Turni et al. 2009). Recently, researchers have reported that the infB gene is useful as a genetic marker for phylogenetic studies. This observation is also true for $H$. parasuis, in that the infB gene be used to distinguish $H$. parasuis from all other closely related species, such as Actinobacillus suis and A. pleuropneumoniae (Hedegaard et al. 2000; Turni et al. 2009). In this study, we evaluated the applicability and efficacy of infB-LAMP for the identification of $H$. parasuis from 187 different specimens. Analysis of these samples by infB-LAMP resulted in the positive identification of $89 \mathrm{H}$. parasuis samples, compared to only 64 positive samples identified by PCR. The results of this study also indicate that $H$. parasuis is widely distributed in South China. Moreover, the infB-LAMP technique has the potential to be applied in clinical settings in which 16S rRNAPCR is too sophisticated to be implemented. In addition, it is a suitable tool for investigating the epidemiology of $H$. parasuis in a clinic.

Detection and isolation of $H$. parasuis is important in understanding the epidemiology of $H$. parasuis infections and can provide critical information used in the design of vaccines specific to strains endemic to certain geographic locations. In this study, the $H$. parasuis serovars present in South China were determined by GD and IHA. These results showed that $32(74.4 \%)$ H. parasuis isolates could be assigned accurately to a specific serovar, whilst nine $(20.9 \%) H$. parasuis serovars were identified and $11(25.6 \%)$ isolates are typically non-typable. So, the PFGE method was used to analyse the genetic diversity of $H$. parasuis isolates collected from South China and a computer analysis program was used to determine similarities between isolates, thereby providing a more objective assessment of clonality (in conjunction with epidemiologic data) that can be used to establish $H$. parasuis as the etiologic agent of animal disease in this region.

\section{Conclusion}

In this study, our results indicate that infB-LAMP can be used in the diagnosis of $H$. parasuis infections, which is essential in defining the exact prevalence of Glässer's disease in South China. Combining this assay with traditional serotyping methods and a highly discriminatory PFGE genotyping technique will provide comprehensive information regarding the epidemiology of $H$. parasuis in South China. Knowledge of the $H$. parasuis strains present in this region can be used in the rational design of vaccines against these isolates, in addition to providing information useful to epidemiologic research.

\section{Acknowledgements}

We would like to thank Professor Huanchun Chen (College of Veterinary Medicine, Huazhong Agricultural University) for providing $15 \mathrm{H}$. parasuis reference strains. This work was supported by grants from the Program for New Century Excellent Talents in University (Grant No. NCET06-0752) and Guangdong Technology Planning Committee (Grant No. 2006B0152, 2009A0201006, 2009B030803050 and 2011B020306010).

\section{Competing interests}

The authors declare that they have no financial or personal relationship(s) which may have inappropriately influenced them in writing this article.

\section{Authors' contributions}

C.X. (South China Agricultural University) was the project leader, H.S. (South China Agricultural University) and J.Z. (South China Agricultural University) were responsible for experimental and project design and M.L. (South China Agricultural University), S.F. (South China Agricultural University) and B.Z. (South China Agricultural University) were in charge of clinical samples collection. L.G. (South China Agricultural University) and J.L. (South China Agricultural University) performed most of the bacterial isolation and PCR detection, whilst H.F. (South China Agricultural University), J.C. (South China Agricultural University) and T.R. (South China Agricultural University) prepared the DNA of the samples, in order to optimise the reaction condition and establish the LAMP.

\section{References}

Aarestrup, F.M., Seyfarth, A.M. \& Angen, $\varnothing$., 2004, 'Antimicrobial susceptibility of Haemophilus parasuis and Histophilus somni from pigs and cattle in Denmark', Veterinary Microbiology 101, 143-146. http://dx.doi.org/10.1016/j. vetmic.2004.02.012, PMid:15172697

Alhassan, A., Thekisoe, O.M.M., Yokoyama, N., Inoue, N., Motloang, M.Y., Mbati, P.A et al., 2007, 'Development of loop-mediated isothermal amplification (LAMP) method for diagnosis of equine piroplasmosis', Veterinary Parasitology 143 155-160.

Angen, $\varnothing$., Oliveira, S., Ahrens, P., Svensmark, B. \& Leser, T.D., 2007, 'Development of an improved species specific PCR test for detection of Haemophilus parasuis', Veterinary Microbiology 119, 266-276. http://dx.doi.org/10.1016/j. vetmic.2006.10.008, PMid:17113728

Baumann, G. \& Bilkei, G., 2002, 'Effect of vaccinating sows and their piglets on the development of Glässer's disease induced by a virulent strain of Haemophilus parasuis serovar 5', Veterinary Record 151, 18-21. http://dx.doi.org/10.1136/ vr.151.1.18, PMid:12137419

Cai, X., Chen, H., Blackall, P.J., Yin, Z., Wang, L., Liu, Z. et al., 2005, 'Serological characterization of Haemophilus parasuis isolates from China', Veterinary Microbiology 111, 231-236. http://dx.doi.org/10.1016/j.vetmic.2005.07.007, PMid:16271834

Hedegaard, J., Hauge, M., Fage-Larsen, J., Mortensen, K.K., Kilian, M., SperlingPetersen, H.U. et al., 2000, 'Investigation of the translation-initition factor IF2gene, infB, as a tool to study the population structure of Streptococcus agalactiae, Microbiology 146, 1661-1670.

Ikadai, H., Tanaka, H., Shibahara, N., Matsuu, A., Uechi, M., Itoh, N. et al., 2004, 'Molecular evidence of infections with Babesia gibsoni parasites in Japan and evaluation of the diagnostic potential of a loop-mediated isothermal amplification method', Journal of Clinical Microbiology 42, 2465-2469. 
Iwamoto, T., Sonobe, T. \& Hayashi, K., 2003, 'Loop-mediated isothermal amplification for direct detection of Mycobacterium tuberculosis complex, M. avium, and M. for direct detection of Mycobacterium tuberculosis complex, M. avium, and M.
intracellulare in sputum samples', Journal of Clinical Microbiology 41, 2616-2622.

Jung, K., Ha, Y., Kim, S.H. \& Chae, C., 2004, 'Development of polymerase chain reaction and comparison with in situ hybridization for the detection of Haemophilus parasuis in formalin-fixed, paraffin-embedded tissues', Journal of Veterinary Medical Science 66, 841-845. http://dx.doi.org/10.1292/jvms.66.841, PMid:15297757

Kuboki, N., Inoue, N., Sakurai, T., Di Cello, F., Grab, D.J., Suzuki, H. et al., 2003, 'Loopmediated isothermal amplification for detection of African trypanosomes' Journal of Clinical Microbiology 41, 5517-5524.

Li, J.X., Jiang, P., Wang, Y., Li, Y.F., Chen, W., Wang, X.W. et al., 2009, 'Genotyping of Haemophilus parasuis from diseased pigs in China and prevalence of two
coexisting virus pathogens', Preventive Veterinary Medicine 91, 274-279. http:// dx.doi.org/10.1016/j.prevetmed.2009.06.004, PMid:19586670

Little, T.W.A., 1970, 'Haemophilus parasuis infection in pigs', Veterinary Record 87, 399-402. http://dx.doi.org/10.1136/vr.87.14.399, PMid:5531588

Minami, M., Ohta, M., Ohkura, T., Ando, T., Torii, K., Hasegawa, T. et al., 2006, 'Use of a combination of brushing technique and the loop-mediated isothermal amplification method as a novel, rapid, and safe system for detection of Helicobacter pylori', Journal of Clinical Microbiology 44, 4032-4037.

Nagamine, K., Hase, T. \& Notomi, T., 2002, 'Accelerated reaction by loop-mediated isothermal amplification using loop primers', Molecular and Cellular Probes 16, 223-229. http://dx.doi.org/10.1006/mcpr.2002.0415, PMid:12144774

Notomi, T., Okayama, H., Masubuchi, H., Masubuchi, H., Yonekawa, T., Watanabe, K. et al., 2000, 'Loop-mediated isothermal amplification of DNA', Nucleic Acids Research 28, E63. http://dx.doi.org/10.1093/nar/28.12.e63

Oliveira, S., Galina, L. \& Pijoan, C., 2001, 'Development of a PCR test to diagnose Haemophilus parasuis infections', Journal of Veterinary Medical Science 13, 495501.

Oliveira, S. \& Pijoan, C., 2004, 'Haemophilus parasuis: New trends on diagnosis, epidemiology and control', Veterinary Microbiology 99, 1-12. http://dx.doi. org/10.1016/j.vetmic.2003.12.001, PMid:15019107
Olvera, A., Segalés, J. \& Aragón, V., 2007, 'Update on the diagnosis of Haemophilus parasuis infection in pigs and novel genotyping methods', Veterinary Journal 174 522-529. http://dx.doi.org/10.1016/j.tvjl.2006.10.017, PMid:17175186

Song, T., Toma, C., Nakasone, N. \& Iwanaga, M., 2005, 'Sensitive and rapid detection of Shigella and enteroinvasive Escherichia coli by a loop-mediated isothermal amplification method', FEMS Microbiology Letters 243, 259-263.

Tadjine, M., Mittal, K.R., Bourdon, S. \& Gottschalk, M., 2004, 'Development of a new serological test for serotyping Haemophilus parasuis isolates and determination of their prevalence in North America', Journal of Clinical Microbiology 24, 839840. http://dx.doi.org/10.1128/JCM.42.2.839-840.2004, PMid:14766867

Thekisoe, O.M.M., Inoue, N., Kuboki, N., Tuntasuvan, D., Bunnoy, W., Borisutsuwan, S. et al., 2005, 'Evaluation of loop-mediated isothermal amplification (LAMP), PCR and parasitological tests for detection of Trypanosoma evansi in experimentally infected pigs', Veterinary Parasitology 130, 327-330.

Tomita, N., Mori, Y., Kanda, H. \& Notomi, T., 2008, 'Loop-mediated isothermal amplification (LAMP) of gene sequences and simple visual detection of products', Nature Protocol 3, 877-882. http://dx.doi.org/10.1038/nprot.2008.57, PMid: Nature Protc

Turni, C., Pyke, M. \& Blackall, P.J., 2009, 'Validation of a real-time PCR for Haemophilus parasuis', Journal of Applied Microbiology 108, 1323-1331. http://dx.doi. org/10.1111/j.1365-2672.2009.04526.x, PMid:19778350

Vanier, G., Szczotka, A., Friedl, P., Lacouture, S., Jacques, M. \& Gottschalk, M., 2006, 'Haemophilus parasuis invades porcine brain microvascular endothelial cells', Microbiology 152, 135-142. http://dx.doi.org/10.1099/mic.0.28312-0, cells', Microbiol 16385123

Wang, Y., Chen, P., Guo, H.B., Chen, Y., Liu, H. \& He, Q.G., 2009, 'Loop-mediated is othermal amplification targeting the apxIVA gene for detection of Actinobacillus othermal amplification targeting the apxIVA gene for detectior
pleuropneumoniae', FEMS Microbiology Letters 300, 83-89.

Zhang, J., Xu, C., Guo, L., Ke, B., Ke, C., Zhang, B. et al., 2011, 'A rapid pulsed-field gel electrophoresis method of genotyping Haemophilus parasuis isolates', Letters in Applied Microbiology 52, 589-595. 